\title{
Fundamental Approach in Defining Optical Crosstalk
}

\author{
Armien John I. Samson
}

\begin{abstract}
In this paper, a more fundamental approach is used in defining optical crosstalk. This is achieved by analyzing the contributions of the non-interacting terms in the probability distribution of detection. Ideally, the detector registers the photons from the source field if and only if an interaction between the source field and the detector occurs. However, there are terms in the probability distribution that suggests that detection occur even when the detector atoms do not interact with the source photons. This is interpreted as the sources of optical crosstalk and it can be argued that these sources of optical crosstalk are due to (a) the secondary photon emission occurring in the bulk of the material which can either be coming directly from the incident photons or from the thermal excitations of the detector atoms; and (b) the interaction of the detector with the vacuum field.
\end{abstract}

Index Terms-Optical crosstalk, optical sensors, photon detection.

\section{INTRODUCTION}

In detection theory, as the sensors receive photon energy (or thermal energy), the incoming photon (or a thermally generated electron-hole pair) can induce secondary emissions during the avalanche breakdown. This secondary emissions in turn causes secondary emissions in the proximity, which then triggers further secondary emissions - thus collectrively introducing noise to the system [1], [2]. The stochastic process that follows is known as optical crosstalk. Because crosstalk introduces unwanted signals in the proximity of the detectors, the system falls into any one or a combination of the following states: a) detection is triggered before actual incoming photon is present (dark count), b) measures higher amplitude in photon detection (after-pulses) [3], or c) in the case of photodetectors, performing sensitive measurements, the presence of such noises can greatly affect the measured output [4].

In practice, the contributions of optical crosstalk to the signal processed by detectors are very hard to ignore and are quite difficult to eliminate. For sources and sensors that lie in the same plane, approaches such as increasing the distance between sensors and detectors as well as placing an optical barrier that eliminates signal from crossing directly into the sensor are employed (see Fig. 1). Numerous configurations have been made by different manufacturers of optical sensors to lower the effects of crosstalk including variations on the geometry of the apertures, applying special ink filters, and optimizing the air gap and glass thickness among other things. While numerous researches on mitigating optical crosstalk were performed on systems that involve an array of

Manuscript received November 15, 2015; revised January 12, 2016. This work was supported in part by ams AG.

A. J. Samson is with the QA Department of ams Asia Inc., he is also with the De La Salle University, Manila, Philippines (e-mail: Armien-John.Samson@ams.com) photodetectors, only a few were devoted in the fundamental understanding of the physical phenomena involved in optical crosstalk.

More importantly, crosstalk is treated from a stochastic point of view where the probabilities of firing of the neighboring detector elements because of a valid detection on one detector element is being modeled using Poisson distribution [5], [6]. A more physical treatment of crosstalk considers the geometrical arrangement of the detector elements involved in order to come up with results signifying local saturation effects and compare the statistical results with experimental data [7]. Instead of taking the statistical route made in various papers, this paper seeks a more fundamental approach to solving the crosstalk problem by developing an analytical model of crosstalk from a quantum mechanical point of view.

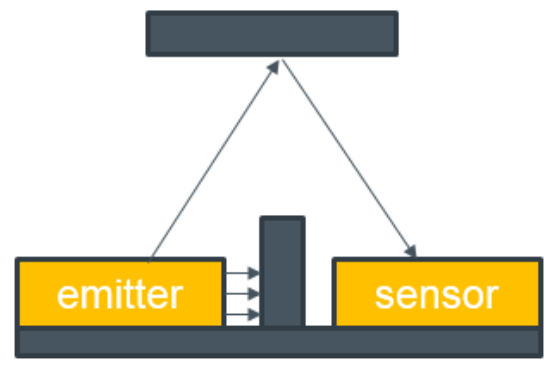

Fig. 1. An optical barrier placed between the emitter and the sensor removes the possibility of crosstalk effect.

\section{PRELIMINARIES}

Crosstalk is phenomenon associated with detection so it is just natural to start with how photons are detected in detectors. Let us consider a collection of many-level atoms localized into point-like photodetector found in the origin and is in ground state $|g\rangle$ with the dipole operator of the detector denoted by $d_{l}$. Let the source dipoles located at some position $r_{j}$ be described by the dipole operator $s_{j}$. Then the interaction Hamiltonian of the detector field and of the source field are given by

$$
\begin{aligned}
& H_{D}=-\sum_{l} d_{l}(t) \cdot E(0, t) \\
& H_{S}=-\sum_{l} s_{j}(t) \cdot E\left(r_{j}, t\right)
\end{aligned}
$$

Here, we would want to understand how these Hamiltonians evolve over time which will then lead to their interaction. The probability distribution of the detection of photon is proportional to the probability of finding a set of $n$ atoms in an excited state, $\left|e_{i}\right\rangle_{l}$, that is

$$
p_{l_{1}, \ldots l_{n}}^{(n)}=\left\langle\varphi_{0}\left|S^{-1}(0, \infty) \hat{P}_{l_{1}, \ldots l_{n}} S(0, \infty)\right| \varphi_{0}\right\rangle
$$


Here, the projection operator $\widehat{P}_{l_{1}, \ldots l_{n}}$ is given by

$$
\hat{P}_{l_{1}, \ldots l_{n}}=\prod_{l=l_{1}}^{l_{n}} \sum_{i}\left|e_{i}\right\rangle_{l l}\left\langle e_{i}\right|
$$

Also, the time evolution operator $S(0, \infty)$ is given by

$$
(0, \infty)=\operatorname{Texp}\left\{\frac{-i}{h} \int_{0}^{\infty}\left(H_{D}(\tau)+H_{S}(\tau)\right) d \tau\right\}
$$

With $T$ being the time ordering given by (and by choosing +1 for bosonic operator for the photons being considered)

$$
\begin{aligned}
& T\left\{A_{1}\left(t_{1}\right) A_{2}\left(t_{2}\right) \ldots A_{n}\left(t_{n}\right)\right\} \\
& =\sum_{p} \theta\left(t_{p 1}>t_{p 2}>\cdots\right. \\
& \left.>t_{p n}\right) A_{p 1}\left(t_{p 1}\right) A_{\cdot p 2}\left(t_{p 2}\right) \ldots A_{p n}\left(t_{p n}\right)
\end{aligned}
$$

In this case, $\theta$ is the Heaviside step function.

The state vector at $t=0$ is considered to be a product of the ground state of the detector atoms, the field vacuum, and some state of the sources, $\left|\psi_{s}\right\rangle$, that is

$$
\left.\left.\left|\varphi_{0}\right\rangle=\prod_{l}\left|g_{l}\right\rangle||\{0\}\right\rangle \psi_{s}\right\rangle
$$

\section{Photon Detection}

Ideally, a photon is detected only when there is an interaction between the photon coming directly from the source and the active area or region of the detector. This means that (4) allows excitation of multiple atoms. At this point, it is very difficult to model this system and so we first consider the probability of detection of a photon by one detector atom - that is, we are looking at the lowest order of coupling of the detector and the source photons. From (4), and if we disregard the vacuum interaction, we see that we are looking for

$$
\begin{aligned}
p_{l}^{1}=\frac{1}{h^{2}} & {\left[\int d t \int d t^{\prime} f_{a b}^{l} G_{a b}\left(0, t \mid 0, t^{\prime}\right) T\right.} \\
& +\int d t \int d \tau f_{a j}^{l} G_{a v}\left(0, t \mid r_{j}, \tau\right) T \\
& \left.+\int d \tau \int d t f_{j a}^{l} G_{v a}\left(r_{j}, \tau \mid 0, t\right) T\right]
\end{aligned}
$$

Here, $f_{a b}^{l}\left(t, t^{\prime}\right)$ is similar to the detector response function defined in [8] where in this case, we have

$$
f_{a b}^{l}\left(t, t^{\prime}\right)=\sum_{a} \sum_{b}\left\langle\varphi_{0}\left|d_{a}(t)\right| e_{i}\right\rangle_{l l}\left\langle e_{i}\left|d_{b}\left(t^{\prime}\right)\right| \varphi_{0}\right\rangle
$$

On the other hand, $G_{a b}$ is the Green's function for many body systems and is given by

$$
G_{a b}\left(\rho, \sigma \mid \rho^{\prime}, \sigma^{\prime}\right)=T^{-1} E_{a}(\rho, \sigma) E_{v}\left(\rho^{\prime}, \sigma^{\prime}\right)
$$

The single time counting probability of the one single detector atom interacting with the field, which is achieved by placing a 'shutter' in front of a detector which is open only for a short time interval $\Delta t$ is given by

$$
\begin{aligned}
& p_{l}^{1}(t, \Delta t) \\
& =\frac{1}{h^{2}}\left[\int_{t}^{t+\Delta t} d t^{\prime} \int_{t}^{t+\Delta t} d t^{\prime \prime} f_{a b}^{l}\left(t, t^{\prime}\right) G_{a b}\left(0, t^{\prime} \mid 0, t^{\prime \prime}\right) T\right. \\
& +\int_{t}^{t+\Delta t} d t^{\prime \prime} \int d \tau f_{a j}^{l}\left(t^{\prime \prime}, \tau\right) v G_{a v}\left(0, t^{\prime \prime} \mid r_{j}, \tau\right) T \\
& \left.+\int d \tau^{\prime} \int_{t}^{t+\Delta t} d t^{\prime} f_{j a}^{l}\left(\tau^{\prime}, t^{\prime}\right) G_{v a}\left(r_{j}, \tau^{\prime} \mid 0, t^{\prime}\right) T\right]
\end{aligned}
$$

Ideally, the detector registers the photons from the source field if and only if an interaction between the source field and the detector occurs, that is for cases whenever $f_{a_{m} j}^{l_{m}}\left(t^{\prime \prime}, \tau\right)$ or $f_{j a_{m}}^{l_{m}}\left(\tau, t_{m}^{\prime}\right)$ is present. In such a case, and if we let

$$
f_{j a_{m}}^{l_{m}}\left(\tau, t_{m}^{\prime}\right)=f_{a_{m} j}^{l_{m}}\left(t_{m}^{\prime \prime}, \tau\right)
$$

Hence, the probability on exciting $l_{n}$ atoms in a short time interval $\Delta t$ is then given by

$$
p_{l_{1}, \ldots l_{n}}^{(n)}=\frac{1}{h^{2}} s_{l}\left(\sigma_{\kappa}, \sigma_{\lambda}\right)
$$

where $s_{l}\left(\sigma_{\kappa}, \sigma_{\lambda}\right)$ takes account the detection of photons from every sources possible (which in this case is valid of the first order perturbation) is given by

$$
\begin{aligned}
& s_{l}\left(\sigma_{\kappa}, \sigma_{\lambda}\right)= \\
& \iint_{A}^{B} d \sigma_{\kappa} d \sigma_{\lambda} \prod_{k=1}^{n} f_{a_{k} b_{k}}^{l_{k}}\left(\sigma_{\kappa}, \sigma_{\lambda}\right) G_{a_{k} b_{k}}\left(r_{\kappa}, \sigma_{\kappa} \mid r_{\lambda}, \sigma_{\lambda}\right) T
\end{aligned}
$$

As we see, (14) follows the following conditions

$$
r_{\kappa} \neq r_{\lambda}=\left\{\begin{array}{lc}
0 & \text { if } \sigma_{\kappa} \in\left(t^{\prime}{ }_{n}, t^{\prime \prime}{ }_{n}\right) \\
r_{j} & \text { otherwise }
\end{array}\right.
$$

With the limits of the integral given by

$$
A, B=\left\{\begin{array}{cc}
t, t+\Delta t & \text { if } \sigma_{\kappa} \in\left(t^{\prime}{ }_{n}, t^{\prime \prime}\right) \\
0, \infty & \text { otherwise }
\end{array}\right.
$$

It is easy to see that (14) means that the probability of detecting a photon coming from the source is equal to the sum of the quantum probabilities that the photons interact with (by exciting the atoms of) the detector field. Because of this, probabilities of detection of photons that do not come directly from the source field is neglected. That is, the photon seen in the first term of (14) as well as the photons from the radiation fields must be neglected as should be the case in an ideal point-like detector, that is

$$
\frac{1}{h^{2}} \int_{t}^{t+\Delta t} d t^{\prime} \int_{t}^{t+\Delta t} d t^{\prime \prime} \prod_{k=1}^{n} f_{a_{k} b_{k}}^{l_{k}}\left(t^{\prime}{ }_{k}, t^{\prime \prime}{ }_{k}\right) G_{a_{k} b_{k}}\left(0, t_{k}^{\prime} \mid 0, t^{\prime \prime}{ }_{k}\right) T=
$$

$$
\begin{gathered}
\frac{i}{h} \int d t \sum_{l}\left\langle\varphi_{0}\left|d_{l}(t)\right| e_{i}\right\rangle_{l l}\left\langle e_{i}\left|T^{-1} E_{a}(0, t) T\right| \varphi_{0}\right\rangle=0 \\
+\frac{i}{h} \int d \tau \sum_{l}\left\langle\varphi_{0}\left|s_{j}^{v}(\tau)\right| e_{i}\right\rangle_{l l}\left\langle e_{i}\left|T^{-1} E_{v}\left(r_{j}, \tau\right) T\right| \varphi_{0}\right\rangle=0
\end{gathered}
$$


In (12), there is an assumption of one-to-one correspondence. That is, each photon coming from the source interacts solely with exactly one atom in the detector which prompts the need to neglect (15). Similarly, we neglect the photons coming from the radiation field by demanding (16) to be zero. The presence of crosstalk in optical sensors and detectors demands that (15) and (16) are no longer zero.

\section{OpticAl CROSSTALK}

The model considered in this case is a point-like detector interacting with the radiation field coming from the point-like source. As can easily be verified in (15) and (16), noises coming from the interaction of the detector with itself as well as noises coming from the interaction of the detector with the vacuum field is present and is an artifact of the perturbative approach in solving the photon detection problem. Hence, we can infer that for the first order perturbation theory, three cases of optical crosstalk can easily be obtained and are outlined in the following:

\section{A. Secondary Emissions of Photons}

Since our detector is point-like, the interaction of the detector dipole with itself which appears in one of the terms of (15) can only mean that a secondary photon has been emitted which was then detected by the detector. This means that (14) will now factor in to (11) and the probability density is changed into

$$
p_{l_{1}, \ldots l_{n}}^{(n)}=\frac{1}{h^{2}} \sum s_{l}\left(\sigma_{\kappa}, \sigma_{\lambda}\right)
$$

With the condition that

$$
r_{\kappa} / r_{\lambda}=\left\{\begin{array}{lc}
0 & \text { if } \sigma_{\kappa} \in\left(t^{\prime}{ }_{n}, t^{\prime \prime}{ }_{n}\right) \\
r_{j} & \text { otherwise }
\end{array}\right.
$$

Equation (18) can be interpreted as the total probability density of detection of photons coming from the detector itself regardless of whether there is an incoming radiation field or not. As we can easily verify, and since we restricted (15) from happening, our detector can still be able to detect photons coming from the detector material itself even when the rest of the terms in (18) is zero. This signal is what we know as the dark count and could arise from the thermal excitations within the detector material, generation of photons due to the presence of significantly strong electromagnetic field in the proximity of the device, and so on.

\section{B. Interaction with the Vacuum Field and the Radiation Field}

Since our detector is point-like, the interaction of the detector dipole with itself can only mean that a secondary photon has been emitted and has been detected by the detector. This means that 14 will now factor in to (11) and the probability density is changed into

$$
p_{l_{1}, \ldots l_{n}}^{(n)}=\frac{1}{h^{2}} \sum s_{l}\left(\sigma_{\kappa}, \sigma_{\lambda}\right)+\frac{i}{h} \sum m_{l}\left(\sigma_{\kappa}\right)
$$

where the detectors interaction with the vacuum field and that of the radiation field is given by

$$
m_{l}\left(\sigma_{\kappa}\right)=\int d \tau \sum_{l} f_{a_{k} 0}^{l_{k}}\left(0, \sigma_{\kappa}\right) G_{a_{k} 0}\left(0, \sigma_{\kappa} \mid 0,0\right) T
$$

Here, the Green's function for multi-body systems and the detector response function takes a different form and are given by

$$
\begin{gathered}
G_{a_{k} 0}\left(0, \sigma_{\kappa} \mid 0,0\right)=\left[T^{-1} E_{v}\left(r_{j}, \tau\right) E_{0}(0,0)\right] \\
f_{a_{k} 0}^{l_{k}}=\left\langle\varphi_{0}\left|s_{j}^{v}(\tau)\right| e_{i}\right\rangle_{l l}\left\langle e_{i}|\{(0)\}| \varphi_{0}\right\rangle
\end{gathered}
$$

\section{Macroscopic Interpretation}

The results obtained in (18) and (19) are idealizations where we assumed that the detector being considered is point-like. In reality, detectors occupy a volume $V$. To bring our results in context, we consider the following. During an infinitesimal time interval $\Delta t$ let a detector of volume $d V=S_{A} d z$ obey the following assumptions:

1) The probability of a single photon detection in that volume is given by

$$
p=\int \eta I(t) d V=\int \eta I(t) S_{A} d z
$$

With $I(t)$ the instantaneous intensity of the incident radiation and $\eta$ is the quantum efficiency of the detector. Here, $\eta$ is given by

$$
\eta=\frac{N_{e}}{N_{v}}
$$

whereby

$$
N_{v}=\frac{\lambda t}{h c} \Phi_{v}
$$

Is the number of the absorbed photon in the depletion layer and

$$
N_{e}=\frac{\lambda t}{h c} \Phi_{e}
$$

Is the number of electrons produced, respectively. Here, $\Phi_{v}$ and $\Phi_{e}$ are the incident optical power (radiant flux) and the optical power absorbed in the depletion layer (both in Watts).

2) The mean number of photons hitting the active area of the detector $S_{A}$ is assumed to be uniformly distributed in space is given by

$$
\left\langle n_{v}\right\rangle=\mathcal{N}_{v} c_{0} \Delta t_{0} S_{A}
$$

where $z_{0}=c_{0} \Delta t_{0}$ is the optical path of the incident photon before hitting the detector and where the number density $\mathcal{N}_{v}$ is defined as

$$
\mathcal{N}_{v}=\frac{d N_{v}}{d V}
$$

Similarly, the mean number of electrons generated from 
these interactions are assumed to be uniformly distributed and is given by

$$
\left\langle n_{e}\right\rangle=\mathcal{N}_{e} c \Delta t S_{A}
$$

where $z=c \Delta t$ is the optical path of the photons interacting with the detector before fully attenuated and where the number density $\mathcal{N}_{e}$ is defined as

$$
\mathcal{N}_{e}=\frac{d N_{e}}{d V}
$$

3) As the incident radiation goes through the volume of the detector, the incident radiation is attenuated. Then, the relationship between the probability of a single photon detection in the given volume and the probability distribution of detection is given by

$$
d p=p_{l_{1}, \ldots l_{n}}^{(1)} d V=p_{l_{1}, \ldots l_{n}}^{(1)} S_{A} d z
$$

Direct comparison between (23) and (28) shows that the probability distribution of detection for a radiant flux incident to the detector in a solid angle is related to the quantum efficiency and the intensity of the radiant flux directed at the detector.

$$
p_{l_{1}, \ldots l_{n}}^{(1)}=\frac{\Phi_{e}}{\Phi_{v}} \frac{d \Phi_{v}}{d \Omega}
$$

Furthermore, if there is no distinction between the time of detection and the generation of electron from the interaction of the photon with the detector, $\Delta t=t$, if we consider a longer interval of the arrival of photons and their detection in such a way that the fluctuations in the number of the arriving photons and absorbed photons within this interval is negligible, and if we consider a small enough optical path for both the incoming photons and the attenuated photons, $z_{0}=z$ then it is easy to show that the probability distribution of detection is also dependent on the index of refraction $n$ of the detector.

$$
p_{l_{1}, \ldots l_{n}}^{(1)}=n\left(\frac{\left\langle n_{e}\right\rangle}{\left\langle n_{v}\right\rangle} \frac{N_{v}}{N_{e}}\right) I
$$

The form taken by the probability distribution of detection in (30) is very interesting. Recall that our problem was to identify the nature of crosstalk and as we have seen in (18) and (30), the probability distribution of detection of crosstalk signals are embedded in the probability of detection of any photon signals. In (30), we see that the quantum mechanical process has a direct relationship with the macroscopic processes involved in the detection of photons. This means that if we can find a way to manipulate or modulate these probabilities in the quantum processes, then we can find ways to completely remove the effect of crosstalk in our detectors. This is particularly promising with the advent of metamaterials and materials where the index of refraction can be changed according to the way we desire. This, however, is not part of this paper and will be considered in the succeeding papers.
From (30), we can infer that the probability of single photon detection in terms of the index of refraction of the material is given by

$$
p=\left(\frac{\left\langle n_{e}\right\rangle}{\left\langle n_{v}\right\rangle} \frac{N_{v}}{N_{e}}\right) S_{A} \int n I d z
$$

Here, (31) assumes that the detector material is linear, homogeneous and isotropic and that the intensity of the radiant flux also varies with the optical length. We also can see that (31) can be modified accordingly to check the probability of detecting a single photon even if the detector material is anisotropic, inhomogeneous, and nonlinear, which also is beyond the scope of this paper.

\section{CONCLUSION}

This paper has two interesting results: First is that it was able to provide a more fundamental relationship involved in the generation of crosstalk by looking at the quantum processes involved in detecting photons. By looking at the time evolution equation and the interactions of the radiation field with the detector, this paper was able to show that optical crosstalk is an artifact of the physical process that takes place, which is as expected. As we have seen, the crosstalk terms are artifacts of the probability distribution of detection and they naturally arise due to the physics behind quantum interactions. In the case of this paper, we have shown that two of the most probable source of crosstalk are (a) the interaction of the detector with the vacuum field and (b) the secondary photon emissions. It is easy to verify that the higher order terms will provide more understanding of other sources of optical crosstalk (like thermal excitations, solitons, etc); Second, this paper is able to make a connection between the quantum process involved in the probability of detecting a photon with the macroscopic process of detection by being able to link the probability distribution of detection to the index of refraction as well as with the intensity of the radiant flux. This is an interesting result since it opens the possibility of developing meta-materials that would purposely remove the introduction of crosstalk in the system, thus eliminating the issue.

\section{ACKNOWLEDGMENT}

The author would like to express his utmost gratitude to ams AG for the support and encouragement. In particular, the author would like to express his gratitude to Alvin Ow for providing valuable advices; for the ams AG Technical Team for their input in the research; and for the ams Calamba for the help they have extended.

\section{REFERENCES}

[1] D. Renker. (April 7, 2009). Advances in solid state photon detectors Journal of Instrumentation. [Online]. Available: http://dx.doi.org/10.1088/1748-0221/4/04/P04004

[2] S. Villa, A. Lacaita, and A. Pacelli, "Photon emission from Hot electrons in silicon," Physics Review Letter, vol. 52, no. 15, pp 10993-10999, 1995.

[3] J. Enderlein and I. Gregor, "Using flourescence life time for discriminating detector after pulsing in flourescence-correlation spectroscopy," Rev Sci. Instrum., vol. 76, 2005.

[4] M. Yokoyama et al., "Performance of multi-pixel photon counters for the T2K near detectors," Nuclear Instruments and Methods in Physics 
Research Section A: Accelerators, Spectrometers, Detectors and Associated Equipment, vol. 622, issue 3, pp. 567-573, 2010.

[5] W. J. Kindt, H. van Zeiji, and Middelhoek, "Optical crosstalk in geiger mode avalanche photodiode array: Modeling, prevention and measurement," in Proc. Solid-State Device Research Conference, 1998.

[6] I. Rech et al., (2008). "Optical crosstak in single photon avalanche diode arays: A new complete model," Opt. Express, vol. 16, issue 12 pp. 8381-8394, 2008.

[7] L. Gallego, J. Rosado, F. Blanco, and F. Arqueros, "Modeling crosstalk in silicon photomultipliers," 2014.

[8] M. Fleischhauer, "Quantum-theory of Photodetection without the rotating wave approximation," Journal of Physics and Math, pp. 453-463, 1998

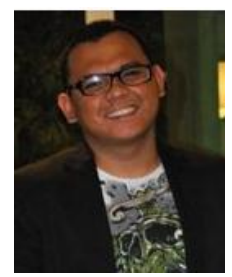

Armien John Samson was born in the Philippines on October 23, 1984. He is currently a candidate for MSc in physics in De La Salle University, Manila, Philippines, working mainly on cloaking and transformation optics.

$\mathrm{He}$ is currently working as a failure analysis engineer at ams Asia in Calamba, Laguna, Philippines. He used to teach IB HL physics and mathematics in Beacon Academy, and was a Sr. failure analysis engineer in international rectifier. 